\title{
Inhibition of HGF/MET signaling decreases overall tumor burden and blocks malignant conversion in Tp/2-related skin cancer
}

\author{
Nicole F. Bonan ${ }^{1}$, David Kowalski ${ }^{1}$, Kaitie Kudlac ${ }^{1}$, Kira Flaherty ${ }^{1}$, J. Curtis Gwilliam¹, Lauren G. Falkenberg', \\ Erik Maradiaga ${ }^{1}$ and Kathleen L. DeCicco-Skinner (iD)
}

\begin{abstract}
Tumor progression locus 2 (Tp/2) is a member of the mitogen-activated protein kinase kinase kinase (MAP3K) family of serine/threonine kinases. Deletion of the Tp/2 gene is associated with a significantly higher number of papillomas and cutaneous squamous cell carcinomas (cSCCs). Overexpression of hepatocyte growth factor (HGF) and its receptor MET is abundant in CSCC and can lead to increased proliferation, migration, invasion or resistance to epidermal growth factor receptor (EGFR) tyrosine kinase inhibitors. The aim of this study was to address whether the increased tumor burden in Tp/2 $2^{-1-}$ mice is due to aberrant HGF/MET signaling. C57BI/6 wild type (WT) and Tp/2 ${ }^{-1-}$ mice were subjected to a two-stage chemical carcinogenesis protocol for one year. At the time of promotion half of the mice received $44 \mathrm{mg} / \mathrm{kg}$ capmatinib (INC 280), a pharmacological inihibitor of MET, in their diet. Tp/2 ${ }^{-1-}$ mice had signficantly higher tumor incidence and overall tumor burden compared to WT mice. Further, carcinogen-intiated Tp/2 ${ }^{-1-}$ mice could bypass the need for promotion, as $89 \%$ of Tp/2 ${ }^{-1-}$ mice given only DMBA developed papillomas. $\mathrm{V}^{-}$ ras $^{\mathrm{Ha}}$-transduced keratinocytes and SCCs from Tp/2 ${ }^{-1-}$ mice revealed an upregulation in HGF and P-MET signaling compared to WT animals. Long-term capmatinib treatment had no adverse effects in mice and capmatinib-fed Tp/2 ${ }^{-1-}$ mice had a $60 \%$ reduction in overall tumor burden. Further, no tumors from Tp/2 ${ }^{-1-}$ mice fed capmatinib underwent malignant conversion. In summary targeting MET may be a potential new strategy to combat cutaneous squamous cell carcinomas that result from dysregulation in MAPK signaling.
\end{abstract}

\section{Introduction}

Cutaneous squamous cell carcinoma ( $\mathrm{CSCC})$ is the second most common form of cancer in the United States and has the highest mortality of all non-melanoma skin cancers ${ }^{1}$. Approximately $2-5 \%$ of cSCC patients develop metastatic disease associated with recurrence and poor long-term prognosis ${ }^{1}$. Skin carcinogenesis is a complex, multistep process and in vivo mouse models remain among the best established tools to study skin cancer development and progression ${ }^{2}$. In the two-stage skin cancer model, initiation often involves application of the carcinogen 7,12-dimethylbenz[a]-anthracene (DMBA),

\footnotetext{
Correspondence: Kathleen L. DeCicco-Skinner (decicco@american.edu) 'Department of Biology, American University, 4400 Massachusetts Ave, NW, Washington, DC 20016, USA
}

which induces an A to $\mathrm{T}$ transversion in codon 61 of the $\mathrm{H}$-Ras gene, followed by repeated applications of the tumor promoter 12-O-tetradecanoylphorbol-13-acetate $(\mathrm{TPA})^{2}$. Tumors begin to develop within a few months, depending on mouse strain, with a small percentage progressing to invasive $\mathrm{SCC}^{2}$.

Tumor progression locus 2 (Tpl2), also called MAP3K8, is a member of the mitogen-activated protein kinase (MAPK) family. Tpl2 has a divergent role in cancer, working as an oncogene or tumor suppressor gene depending on context or tissue in which the signal is aberrantly altered ${ }^{3}$. Increased $T p l 2$ activity has been reported in multiple cancer types, including breast, colon and gastric cancer, nasopharyngeal carcinoma, thymoma, lymphoma, and keratoacanthoma ${ }^{4-9}$. However, Tpl2 can 
function as a tumor suppressor in cancers such as cSCC, lung cancer, colitis-associated tumorigenesis, and $\mathrm{T}$-cell lymphomas, suggesting the role of $T p l 2$ in carcinogenesis remains unclear ${ }^{10-14}$. In a DMBA/TPA carcinogenesis model, we have previously shown that mice devoid of $T p l 2$ have a significantly higher incidence of skin tumor development, associated with increased inflammatory signaling and epithelial proliferation ${ }^{11,14,15}$. Additionally, we found stromal fibroblasts contribute to this tumorigenesis.

Hepatocyte growth factor (HGF) is a key factor in the malignant crosstalk between stromal fibroblasts and the primary tumor ${ }^{16}$. HGF released from fibroblasts acts as a ligand for a tyrosine kinase receptor, MET, on neighboring tumor cells. Following ligand binding and autophosphorylation of MET, a variety of signaling pathways become activated including PI3K/AKT, RAS/MAPK, STAT3, WNT, and $\mathrm{NFKB}^{16}$. In addition to HGF/MET paracrine signaling, HGF and MET can be co-expressed in many types of cancers, producing an autocrine loop ${ }^{16}$.

Activation of HGF/MET signaling can contribute to proliferation, survival, invasion, migration, drug resistance, and/or angiogenesis in a wide variety of tumors, and correlates with poor clinical outcome ${ }^{17}$. Abundant MET expression or increased HGF transcript and protein levels are found in human skin $\mathrm{SCC}^{18-20}$. Aberrant MET activation can occur through several mechanisms including autocrine/paracrine HGF stimulation, mutational activation, gene amplification or upregulation $^{16,17,21}$.

Because HGF and MET are both implicated in cancer progression, many inhibitors have been developed as targeted cancer therapies, including MET inhibitors, antiMET monoclonal antibodies, and anti-HGF antibodies ${ }^{22}$. Capmatinib (INC280 or INCB28060) is a highly selective, oral small molecule kinase inhibitor of $\mathrm{MET}^{23}$. Capmatinib has shown effectiveness in vitro as a single or combined agent in a variety of cancers ${ }^{24-30}$. It is currently in phase I/II clinical trials for papillary renal cancer, hepatocarcinoma (HCC), non-small cell lung cancer (NSCLC), and other advanced solid tumors, and has shown some clinical efficacy ${ }^{31,32}$. However, to our knowledge capmatinib has not been tested for its ability to block skin tumor formation or malignant conversion of chemically-induced papillomas.

In the current study we sought to identify if dysregulation in HGF/MET signaling is responsible for the increase in tumor formation and progression in mice devoid of Tpl2. We found an overexpression of HGF/ MET signaling in $\mathrm{v}$-ras ${ }^{\mathrm{Ha}}$-transduced keratinocytes and SCCs from Tpl2 ${ }^{-/-}$mice. Pharmacological inhibition of MET by capmatinib could decrease overall tumor burden in Tpl2 ${ }^{-1-}$ mice by $60 \%$ and prevent all malignant con- version of benign papillomas to invasive SCC. Unexpectedly, DMBA alone was sufficient for tumor formation in Tpl $2^{-1-}$ mice, bypassing the need for a promoter. In summary, heightened HGF/MET signaling is critical for tumor formation and progression in $\mathrm{Tpl} 2^{-/-}$mice.

\section{Results}

\section{Fibroblast signaling contributes to the hyperproliferative phenotype and in vitro conversion frequency of $\mathrm{v}^{-\mathrm{ras}^{\mathrm{Ha}}}{ }_{-}$ transduced keratinocytes}

Stromal fibroblasts play a critical role in the growth and progression of SCC. To assess whether Tpl2 ${ }^{-1-}$ fibroblasts could potentiate growth of $\mathrm{v}_{\text {-ras }}{ }^{\mathrm{Ha}}$-transduced keratinocytes a proliferation assay was conducted (Fig. 1a). Under normal conditions $v$-ras ${ }^{H a}$-transduced $T p l 2$ ${ }^{-l-}$ keratinocytes grew $44 \%$ faster than $\mathrm{v}^{- \text {ras }^{H a}}{ }^{\mathrm{Ha}}$-transduced WT keratinocytes (Fig. 1a; $p<0.01$ ). Additionally, $v$-ras ${ }^{H a}$-transduced WT keratinocytes grown in $T p l 2^{-1-}$ fibroblast conditioned media had a $71 \%$ increase in cell proliferation compared to $v$-ras ${ }^{H a}$-transduced WT keratinocytes grown in normal supplemented media. $\mathrm{v}_{\text {-ras }} \mathrm{Ha}_{\text {- }}$ transduced $T p l 2^{-1-}$ keratinocytes grown in $T p l 2^{-1-}$ fibroblast conditioned media had a $32 \%$ increase in cell proliferation compared to $v$-ras ${ }^{H a}$-transduced $T p l 2^{-1-}$ keratinocytes grown in normal supplemented media (Fig. 1a; $p<0.01$ ).

To determine whether fibroblast signaling could induce spontaneous, hyperproliferative, neoplastic growth in keratinocytes, an in vitro conversion assay was conducted. Keratinocytes were infected with v-ras ${ }^{\mathrm{Ha}}$ retrovirus. Cells that don't incorporate $\mathrm{v}$-ras ${ }^{\mathrm{Ha}}$ retrovirus undergo terminal differentiation in high calcium media. The remaining cells can stay as a monolayer or have the potential to form proliferative foci. $v$-ras ${ }^{H a}$-transduced WT keratinocytes developed no foci by 8 weeks when grown in normal supplemented high calcium DMEM media (Table 1, Fig. $1 \mathrm{~b}$, "i"). However, when WT cells were grown in WT fibroblast conditioned media for 8 weeks $60 \%$ of the cultures developed a focus and when WT cells were grown in $T p l 2^{-1-}$ fibroblast conditioned media $40 \%$ of the cultures developed a focus. However, this fell short of achieving statistical significance (Table 1). $v$-ras ${ }^{\mathrm{Ha}}$ -transduced Tpl2 $2^{-1-}$ keratinocytes grown in normal supplemented high calcium DMEM media developed no foci by 8 weeks (Table 1, Fig. 1b "ii"). However, $83 \%$ of the $\mathrm{Tpl} 2^{-1-}$ cultures developed a single focus in response to signals from WT fibroblasts (Table 1, Fig. 1b, "v", $p<$ 0.005 ) and $100 \%$ of the Tpl2 ${ }^{-1-}$ keratinocyte cultures grown in conditioned media from $T p l 2^{-1-}$ fibroblasts developed at least one focus, with one culture developing two foci (Table 1, Fig. 1b "vi", $p<0.00001$ ). Rhodamine staining was used to differentiate the foci in the cultures from cells in a monolayer. 
A

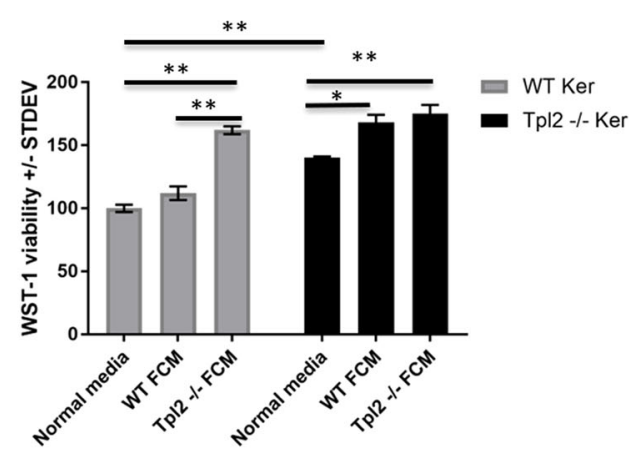

C

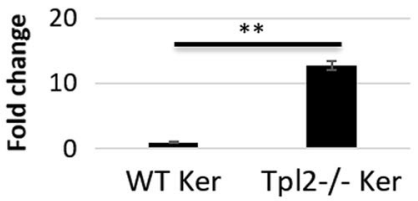

F

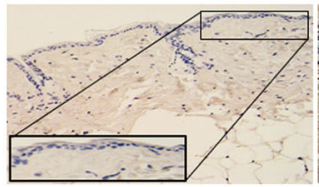

WT Skin
D

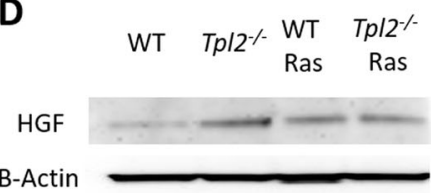

G

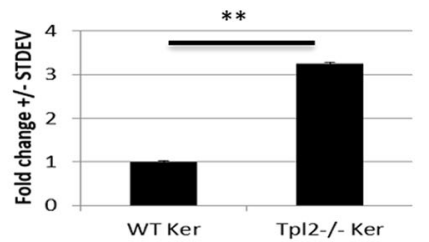

B

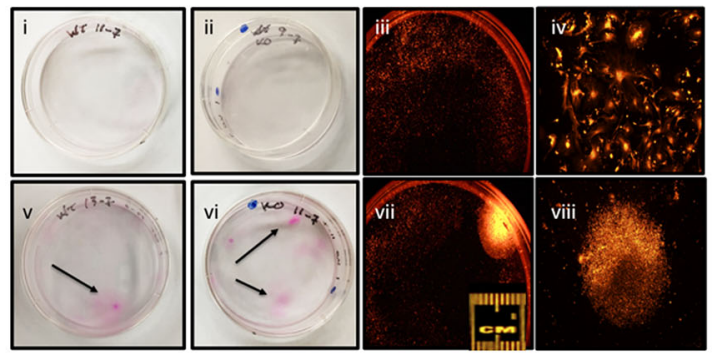

E

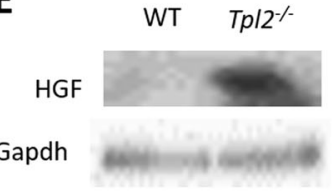

H

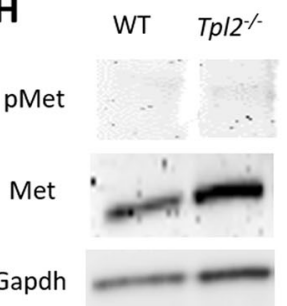

Fig. $1 \mathrm{Tp} / 2^{-/-}$keratinocytes have an increased cell cycle, propensity for malignant conversion, and HGF and MET expression compared to Tpl2 $^{+/+}$(WT) cells. a WST-1 viability assay for -ras $^{\text {Ha }}$-transduced Tpl2 ${ }^{-/-}$and WT keratinocytes (RAS) grown in conditioned media from Tpl2 ${ }^{-/-}$or WT fibroblasts. Results normalized to the $\mathrm{v}$-ras $^{\mathrm{Ha}}$-transduced Tp/2 ${ }^{+/+}$(WT) keratinocyte control. ${ }^{*} p<0.05,{ }^{* *} p<0.01$. $\mathbf{b}$ v-ras ${ }^{\mathrm{Ha}}$-transduced WT and $\mathrm{Tpl}^{-1-}$ keratinocyte malignant conversion in response to fibroblast signaling. $\mathrm{v}$-ras ${ }^{\mathrm{Ha}}$-transduced WT ("i") or Tpl2 ${ }^{-/-}$("ii") keratinocytes cultured in high calcium media develop no foci and remain quiescent in a dispersed monolayer which can be seen at 4 X ("iii") and 40X ("iv") magnification using rhodamine staining. WT (" $\mathrm{v}^{\prime)}$ ) and Tpl2 ${ }^{-/-}($"vi") keratinocytes cultured in high calcium fibroblast conditioned media form proliferative foci. Foci from rhodamine-stained cultures stain brightly ("vi", 4X magnification; "vii", 40X magnification) under fluorescence microscopy. Real-time PCR quantification of HGF in keratinocytes (c) and immunoblotting (d) of HGF in untreated and v-ras ${ }^{\text {Ha }}$-transduced Tpl $2^{-1-}$ and WT keratinocytes. Immunoblotting of HGF in fibroblasts (e) and immunostaining of HGF in skin (f). Real-time PCR quantification (g) and immunoblotting (h) of p-MET. ${ }^{*} p<0.05$

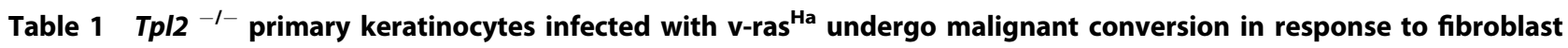
signals

\begin{tabular}{llll}
\hline Condition & Foci & Ratio of conversion & Significance \\
\hline WT Ker Ras & 0 & $0 / 5$ & NS \\
WT Ker Ras in WT FCM & 3 & $3 / 5$ & NS \\
WT Ker Ras in KO FCM & 2 & $2 / 5$ & $P<0.005$ (vs. KO Ker Ras) \\
KO Ker Ras & 0 & $0 / 6$ & $5 / 6$ \\
KO Ker Ras in WT FCM & 5 & $7 / 6$ & $P<0.000001$ (vs. KO Ker Ras) \\
KO Ker Ras in KO FCM & 7 & &
\end{tabular}

HGF and MET are upregulated in $\mathrm{Tpl} 2^{-1-}$ cells

Overexpression of $\mathrm{HGF}$ and/or MET can promote tumorigenesis and metastasis. Tpl $2^{-/-}$keratinocytes had twelve-fold higher HGF gene expression (Fig. 1c; $p<0.01$ ) and five-fold higher protein levels $(p<0.05$; Fig. 1d) when compared to WT keratinocytes, although WT and Tpl2 

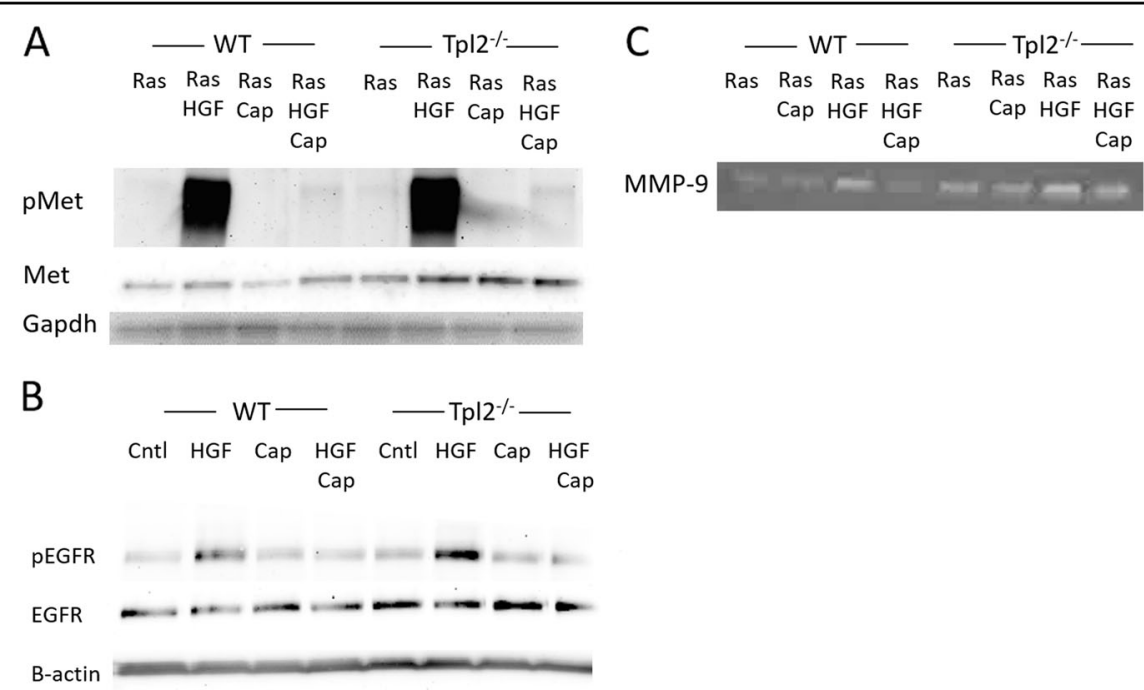

Fig. 2 Capmatinib blocks HGF-mediated p-MET expression, reduces MMP-9 activity and prevents phosphorylation of p-EGFR. Primary keratinocytes from newborn mice were cultured in low calcium $(0.05 \mathrm{mM})$ growth media and transduced with $v$-ras ${ }^{\mathrm{Ha}}$. Cultures were treated with $0.1 \mathrm{nM}$ Capmatinib, followed by administration of $20 \mathrm{ng} / \mathrm{ml} \mathrm{HGF}$ or vehicle. Total protein was lysed 15 min post HGF treatment. Immunoblotting of p-MET/MET and MET (a) p-EGFR/EGFR (b) in total cell extracts from primary control keratinocytes was conducted. Lower exposure of (Fig. 2a) is also shown in Supplementary data B. c Gelatin zymography of MMP-9 activity in conditioned media from v-ras ${ }^{H a}$-transduced Tpl $2^{+/+}$(WT) and Tpl2 ${ }^{-/-}$ (KO) keratinocytes treated with $\mathrm{HGF}+/$ - Capmatinib

${ }^{-1-}$ keratinocytes that had been transduced with $v$-ras ${ }^{H a}$ retrovirus had comparable levels. Fibroblasts from $T p l 2$ $-{ }^{-}$mice had 8.7 -fold higher HGF protein $(p<0.05)$ compared to WT fibroblasts (Fig. 1e). Additionally, HGF was 2.5 fold higher in skin of Tpl2 ${ }^{-1-}$ mice compared to WT mice (Fig. 1f; $p<0.05$ ).

MET transcripts were 3.1-fold higher in Tpl2 ${ }^{-1-}$ keratinocytes compared to WT cells (Fig. 1g; $p<0.01$ ). pMET was undetectable in unstimulated WT keratinocytes and barely detectable in $\mathrm{Tpl} 2^{-/-}$keratinocytes. In unstimulated conditions total MET protein was 1.8 fold higher in $\mathrm{Tpl}^{-1-}$ keratinocytes (Fig. 1h). In v-ras ${ }^{\mathrm{Ha}}$-transduced keratinocytes HGF stimulation increased p-MET 27 fold in WT keratinocytes and 36 fold in Tpl2 $2^{-l-}$ cells (Fig. 2a, Supplementary data A, Supplementary data B) compared to unstimulated $\mathrm{v}$-ras ${ }^{\mathrm{Ha}}$-transduced keratinocytes $(p<$ $0.01)$. Moreover, total MET protein was 2-fold higher in $\mathrm{Tpl} 2^{-/-}$keratinocytes transduced with $\mathrm{v}$-ras ${ }^{\mathrm{Ha}}$ -retrovirus (Fig. 2a).

\section{Capmatinib blocks MET phosphorylation, MMP-9 activity, and p-EGFR phosphorylation}

Capmatinib is a small molecule kinase inhibitor of MET. WT and Tpl2 ${ }^{-1-}$ keratinocytes were treated with HGF, Capmatinib, or HGF + Capmatinib and protein extracted to confirm the in vitro effectiveness of this drug. Capmatinib inhibited the HGF-stimulated p-MET at concentrations as low as $0.1 \mathrm{nM}$ (Fig. 2a). Recent evidence suggests that MET can interact with EGFR in the $\operatorname{skin}^{20}$.
Therefore, we tested whether blocking MET phosphorylation with capmatinib can also block p-EGFR. Tpl2 ${ }^{-1-}$ keratinocytes had two-fold higher p-EGFR compared to WT keratinocytes, both under basal conditions and when stimulated with HGF (Fig. 2b). Treatment with capmatinib fully blocked the HGF-mediated increase in p-EGFR, restoring levels to baseline conditions, yet had no effect on total EGFR protein (Fig. 2b).

Upregulation in MET can increase Matrix Metalloproteinase 9 (MMP-9) production by tumor-associated stromal cells, and blocking MET can significantly reduce MMP-9 activity ${ }^{33,34}$. Thus, we measured MMP-9 activity in $v$-ras ${ }^{\mathrm{Ha}}$-transduced WT and Tpl2 ${ }^{-1-}$ keratinocytes. MMP-9 activity was 2.3 fold higher in conditioned media from $v$-ras ${ }^{\mathrm{Ha}}$-transduced $\mathrm{Tpl} 2^{-1-}$ keratinocytes compared to $v$-ras ${ }^{H a}$-transduced WT cells both under basal conditions and when stimulated with HGF. (Fig. 2c). Capmatinib treatment decreased HGF-induced MMP-9 activity in WT keratinocytes 4-fold and Tpl2 ${ }^{-/-}$keratinocytes two-fold.

\section{$\mathrm{Tpl} 2^{-1-}$ primary keratinocytes are refractory to TGF $\beta$} mediated growth inhibition despite having normal levels of TGF $\beta$ receptors and SMAD proteins

In some cancers a defect in TGF $\beta$ signaling can result in overexpression of $\mathrm{HGF}^{35}$. To assess whether the increase in HGF signaling in $T p l 2^{-1-}$ keratinocytes correlates with a defect in TGF $\beta$ signaling we measured levels of TGF $\beta 1$, TGF $\beta R$ I and II, and associated SMAD proteins (Fig. 3a, 

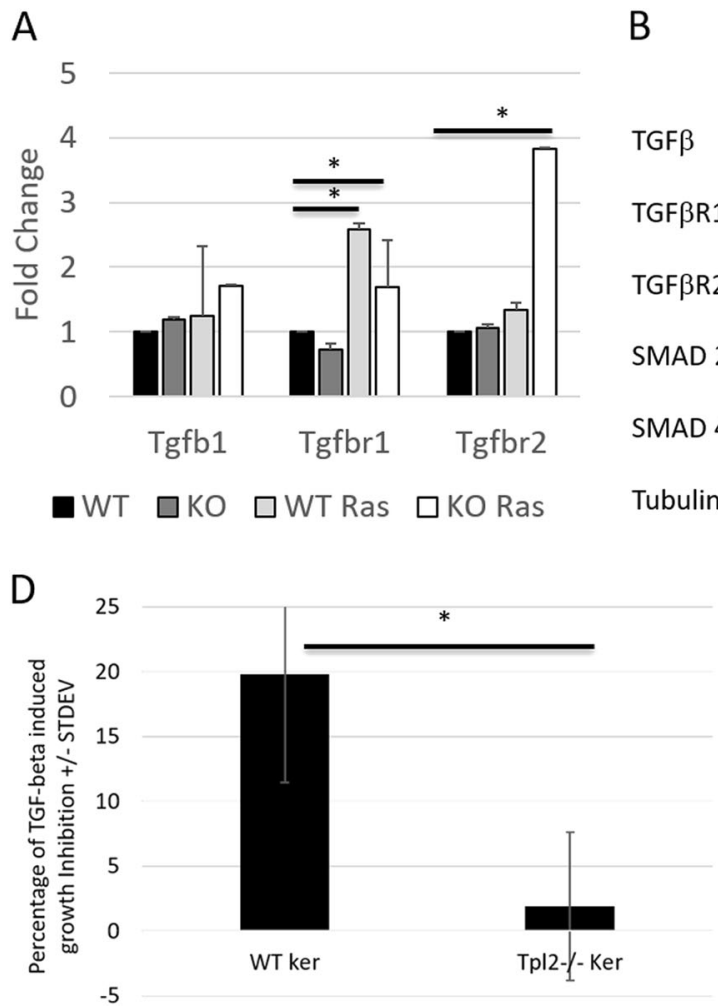

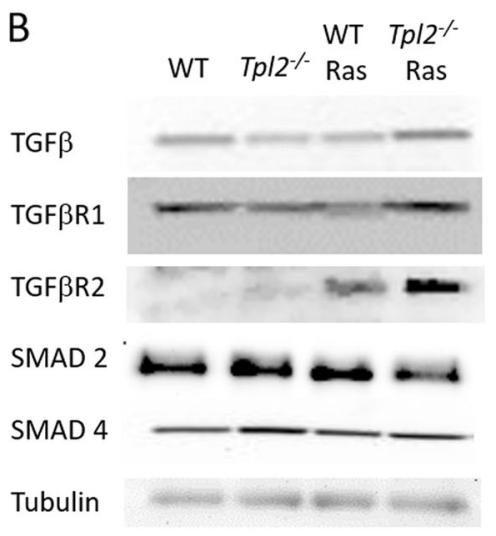

C

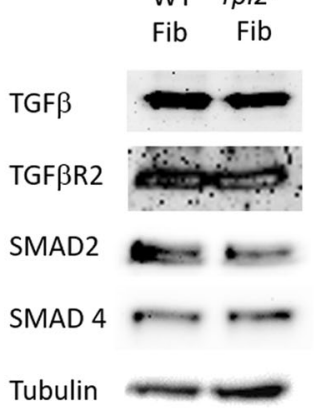

$\mathrm{E}$

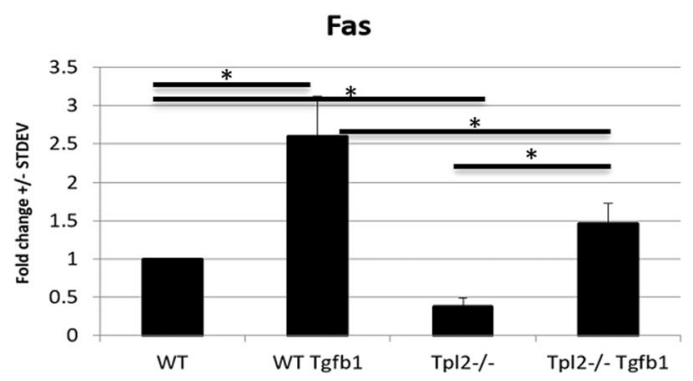

Fig. $3 \mathrm{Tpl}^{-1-}$ primary keratinocytes are refractory to TGF $\beta$ mediated growth inhibition despite having normal levels of TGF $\beta$ receptors and SMAD proteins. Primary keratinocytes were cultured in low calcium $(0.05 \mathrm{mM})$ growth media or transduced with $v$-ras ${ }^{\text {Ha }}$ (RAS). Real-time PCR (a) and Western analyses (b) were conducted for TGFB, TGFBRI and TGFBRII, SMAD2 and SMAD4. c Fibroblast protein was collected from newborn WT and Tpl2 $2^{-1-}$ mice and used for Western analysis. All expression was normalized to a housekeeping gene. $\mathbf{d}$ WT and Tpl2 $2^{-1-}$ keratinocytes were treated with $10 \mathrm{ng} / \mathrm{ml}$ TGF $\beta$ and after $24 \mathrm{~h}$, proliferation was determined using a WST-1 assay. e Whole mRNA was isolated and real-time PCR performed and analyzed for relative abundance of Fas mRNA

b). While TGF $\beta 1$ protein levels were 2-fold lower in Tpl2 ${ }^{-1-}$ keratinocytes $(p<0.05)$, there was no significant defect in TGF $\beta$ R I and II, SMAD 2 or SMAD 4 proteins in keratinocytes or fibroblasts. (Fig. 3b, C) Interestingly, v$\mathrm{ras}^{\mathrm{Ha}}$-transduced $\mathrm{Tpl} 2^{-1-}$ keratinocytes had an increase in TGF $\beta$, TGF $\beta$ RI and TGF $\beta$ RII gene expression (Fig. 3a, $p<0.01$ ) and ten-fold higher TGF $\beta$ RII protein levels compared to untreated keratinocytes.

TGF $\beta$ is a potent inhibitor of proliferation in most cell types $^{36}$. Thus, we assessed the ability of TGF $\beta$ to inhibit proliferation of WT and Tpl2 ${ }^{-1-}$ keratinocytes. Within $24 \mathrm{~h}$ WT keratinocytes exhibited a $20 \%$ decrease in proliferation in response to TGF $\beta$, while $T p l 2^{-1-}$ keratinocytes exhibited no growth inhibition (Fig. $3 \mathrm{~d} ; p<0.05$ ). The apoptosis-inducing protein Fas is typically overexpressed in TGF $\beta$-stimulated cells, as it participates in TGF $\beta$ induced growth arrest. Tpl2 ${ }^{-1-}$ keratinocytes exhibit decreased transcription of Fas relative to wild-type keratinocytes, both when grown under basal conditions and when grown in the presence of TGF $\beta$ (Fig. 3e; $p<$ 0.05)

\section{Pharmacological inhibition of MET decreases skin tumor} formation in $\mathrm{Tpl}^{-/-}$mice

To determine if upregulation of HGF/MET signaling contributes to an increased incidence of tumor formation in Tpl2 ${ }^{-1-}$ mice, a two-stage chemical carcinogenesis model was employed. Hundred percent of $T p l 2^{-1-}$ mice subjected to two-stage chemical carcinogenesis developed skin tumors, compared to $58 \%$ of WT animals (Fig. 4a). The tumor latency was significantly $(p<0.002)$ different between genotypes by week 13 , with $63 \%$ of $T p l 2^{-1-}$ mice developing papillomas at week 13 in comparison to $8 \%$ of WT mice. Further, the number of papillomas was strikingly different between genotypes (Fig. $4 \mathrm{~b} ; p=0.001$ ). $\mathrm{Tpl} 2^{-1-}$ mice on normal diet averaged nearly 8 papillomas/mouse and the WT averaged 1.4 tumors/total number of DMBA/TPA WT mice (or 2.28 tumors/mouse if analysis only includes tumor-bearing WT mice). Tpl2 ${ }^{-1-}$ mice fed a capmatinib diet had a $60 \%$ reduction in tumor burden, averaging 3 papillomas/ Tpl $2^{-1-}$ mouse (Fig. 4b and Fig. 4f; $p=0.02$ ). As expected, no WT control 

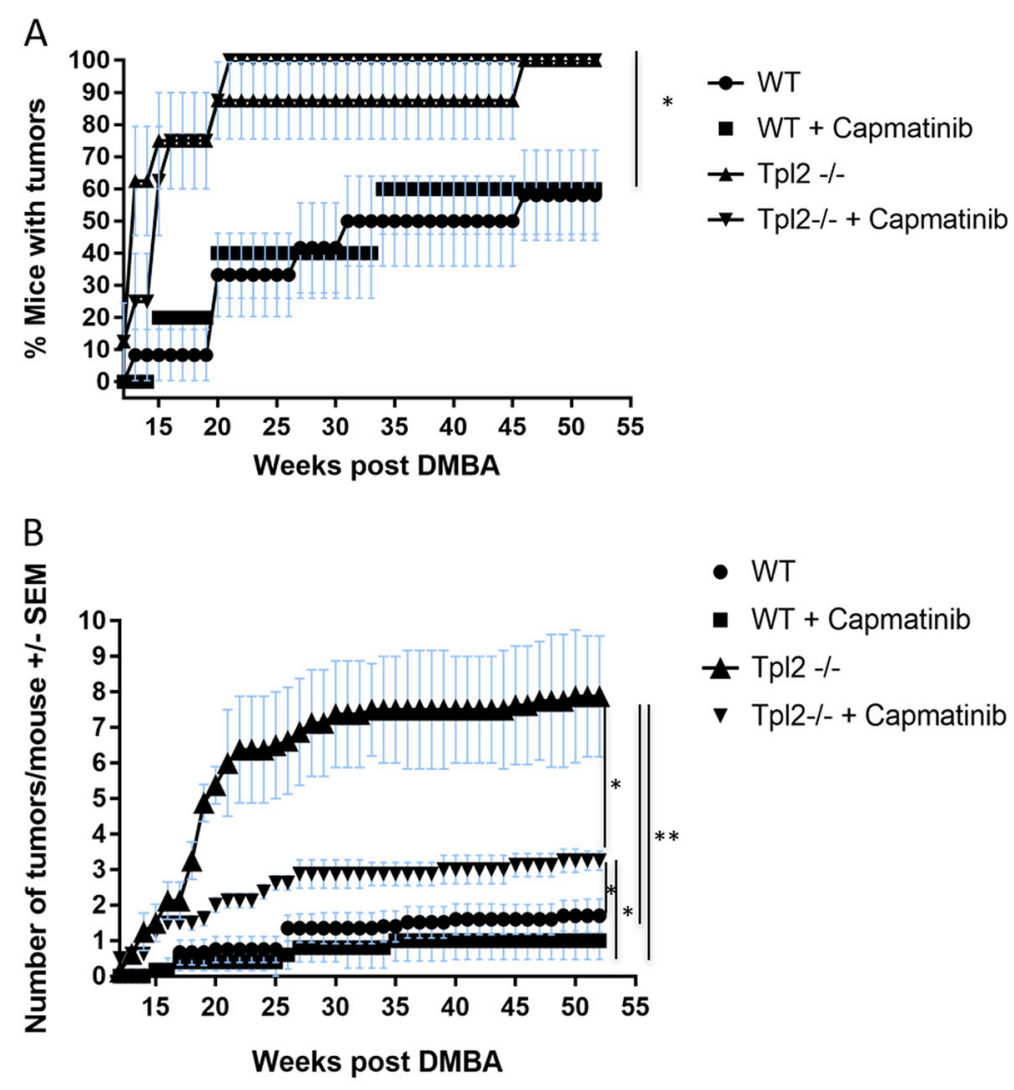

- WT

- $\mathrm{WT}+$ Capmatinib

- Tpl2 - -

> Tpl2-/-+ Capmatinib

C

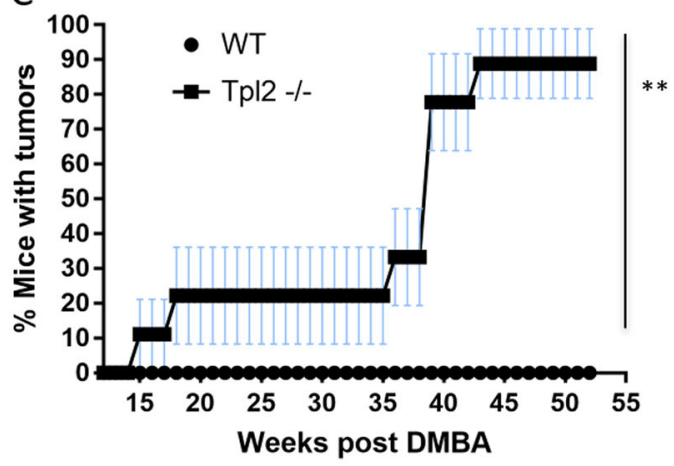

E

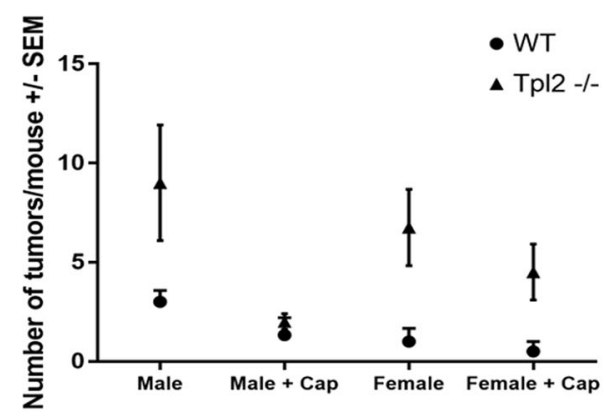

D

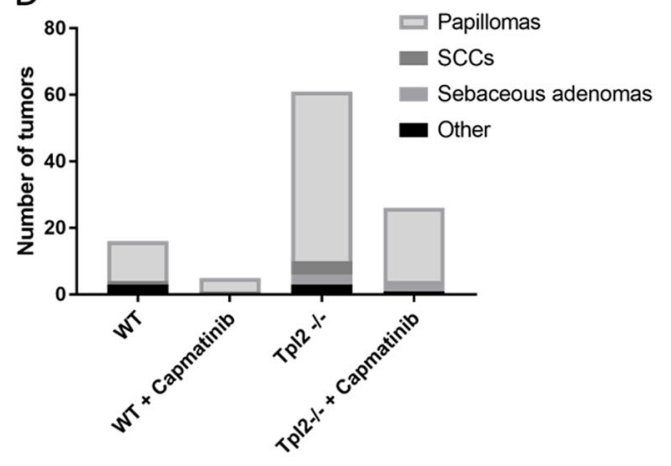

F

F ${ }_{\text {WT }} \quad \mathrm{WT}+$ Cap $\quad \mathrm{Tpl2}^{-}-\quad \mathrm{Tpl}^{2}++$ Cap

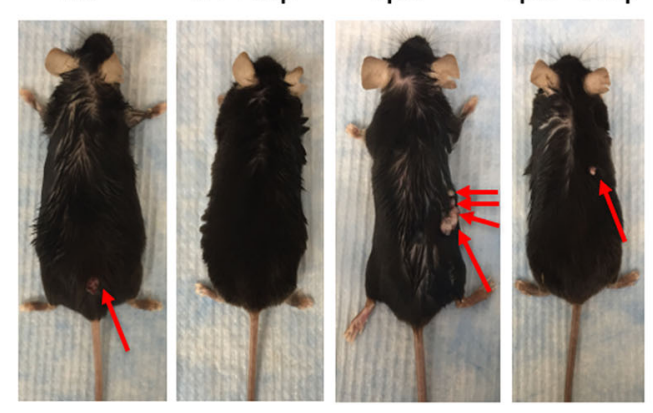

Fig. 4 (See legend on next page.) 
Fig. 4 Capmatinib blocks tumor formation and malignant conversion in $\mathrm{Tpl}^{-1-}$ mice subjected to two-stage chemical carcinogenesis.

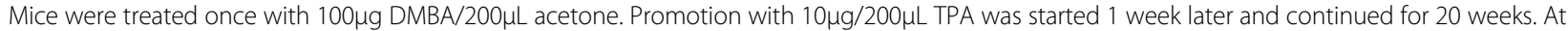
the time of promotion half of each genotype received $44 \mathrm{mg} / \mathrm{kg}$ capmatinib in their diet. Tumors were measured weekly. The percentage of tumorbearing mice (a) and the number of tumors per mouse (b) are plotted vs. time for each group. One-proportion confidence intervals (a) and SEM (b) were used to generate error bars. Significant differences between curves appeared by week 20 and remained significant until the termination of the study. Significant differences were determined by two-way ANOVA $(p<0.001)$. c The percentage of mice receiving DMBA only (no promotion with TPA) are plotted vs. time and significant by week $20(p<0.05)$ and highly significant $(p<0.0001)$ by week 40 . Error bars were calculated using oneproportion confidence intervals. d Summary of histological examination from the two-stage carcinogenesis experiment. e Tumor number and responsivity to capmatinib in males vs. females. $\mathbf{f}$ Photograph of representative DMBA/TPA mice

mice receiving only acetone, TPA or DMBA developed tumors. In a similar fashion no acetone-only or TPA-only Tpl2 ${ }^{-1-}$ mice developed tumors. However, $88.9 \%$ of $T p l 2$ -I- mice treated only with DMBA developed a papilloma on their skin (Fig. 4c; $p<0.0001$ ).

\section{Capmatinib prevents malignant conversion in $\mathrm{Tpl}^{-1-}$ mice}

Tumors underwent a histological examination by a pathologist to determine phenotype and progression. Of the WT mice fed normal diet there were a total of 16 tumors. Twelve of the tumors were papillomas, with one converting to a squamous cell carcinoma (Fig. 4d). Three additional tumors were cutaneous lipomas. This is in comparison to Tpl2 ${ }^{-1-}$ mice which had a total of 61 tumors, 51 papillomas, four SCCs, three sebaceous adenomas, and three lipomas. In contrast, no Tpl $2^{-1-}$ mice fed capmatinib diet had papillomas convert to SCCs (Fig. 4d). Although $T p l 2^{-1-}$ mice develop an overall higher tumor burder, there were no statistical differences in tumor size between genotypes and the rate of malignant conversion (7.8 vs. $8.3 \%$ ) was similar between $T p l 2^{-1-}$ and WT mice on normal diet. However, the rate of malignant conversion between $\mathrm{Tpl} 2^{-1-}$ mice on normal $\operatorname{diet}(8.3 \%)$ vs. $T p l 2^{-1-}$ mice on Capmatinib diet (0\%) was significantly different $(p<0.01)$. In both genotypes male mice developed more tumors than female mice (Fig. $4 \mathrm{e} ; p$ $<0.05)$.

\section{SCCs from Tpl2 ${ }^{-1-}$ mice have increased expression of HGF and $\mathrm{p}-\mathrm{MET}$}

We measured HGF and p-MET expression in papillomas and SCCs from WT and Tpl2 ${ }^{-1-}$ mice. HGF (Fig. 5a) and p-MET (Fig. 5b) levels did not differ statistically between WT and Tpl2 $2^{-1-}$ papillomas. However, both HGF and p-MET expression was significantly $(p<0.05)$ elevated in SCCs of Tpl2 ${ }^{-1-}$ mice compared to WT SCCs (Fig. 5a, b). Additionally, papillomas from mice fed capmatinib diet had a reduction in p-MET levels (Fig. 5b). Expression of p-MET in SCCs from capmatinib-fed Tpl2 ${ }^{-/-}$mice could not be measured, as no Tpl2 ${ }^{-/-}$mice fed capmatinib had papillomas convert to SCCs.

\section{Discussion}

The incidence of cutaneous SCC has sharply risen over the last two decades; thus, so has the need to identify driver mutations and aberrantly regulated signaling pathways. MAPK signaling is frequently overexpressed in SCC, therefore the use of kinase inhibitors as potential anti-cancer agents has been attractive. However, inhibition of MAPK signaling has often led to complicated and unexpected findings such as development of de novo mutations and activation of MAPK-dependent and independent bypass pathways, leading to epithelial proliferation, development of squamous cell carcinomas and/or drug resistance ${ }^{11,37,38}$.

We have previously reported that a MAP3K family member, Tpl2, can work as a tumor suppressor gene in skin, as Tpl $2^{-1-}$ mice develop a significantly higher number of papillomas and $\mathrm{CSCCs}^{11,14,15}$. Contributing to this carcinogenesis we have found that stromal fibroblasts have a marked influence on the hyperproliferative phenotype and angiogenic capabilities of $v$-ras ${ }^{\mathrm{Ha}}$-transduced keratinocytes $^{11,15}$. Fibroblasts secrete a variety of signals, including the growth factor HGF, that are integral to the development and progression of SCC. We measured expression of HGF in keratinocytes, fibroblasts, skin and tumor sections from $T p l 2^{-/-}$mice and found it to be significantly higher than WT controls. This is in agreement with others who reported an increase in HGF production in Tpl2-deficient intestinal myofibroblasts ${ }^{12}$.

HGF, when bound to MET, employs several mechanisms to induce cell migration and invasion ${ }^{17}$. One such mechanism is induction of matrix metalloproteinases such as MMP-9 ${ }^{39}$. We found considerably higher MMP-9 activity in conditioned media from Tpl2 ${ }^{-1-}$ keratinocytes, both in untreated $v$-ras ${ }^{\mathrm{Ha}}$-transduced keratinocytes and upon stimulation with HGF. In gastic cancer cells HGF can induce MMP-9 through upregulation in lipocalin- 2 and activation of $\mathrm{NF}^{3} \mathrm{~B}^{39}$. Notably both of these factors have been previously shown by our laboratory to be elevated in $T p l 2^{-1-}$ keratinocytes ${ }^{11,40}$.

The HGF gene is subject to both positive and negative regulation. Defects in TGF $\beta$ signaling can cause an overproduction of HGF, contributing to tumorigenesis ${ }^{35}$. 


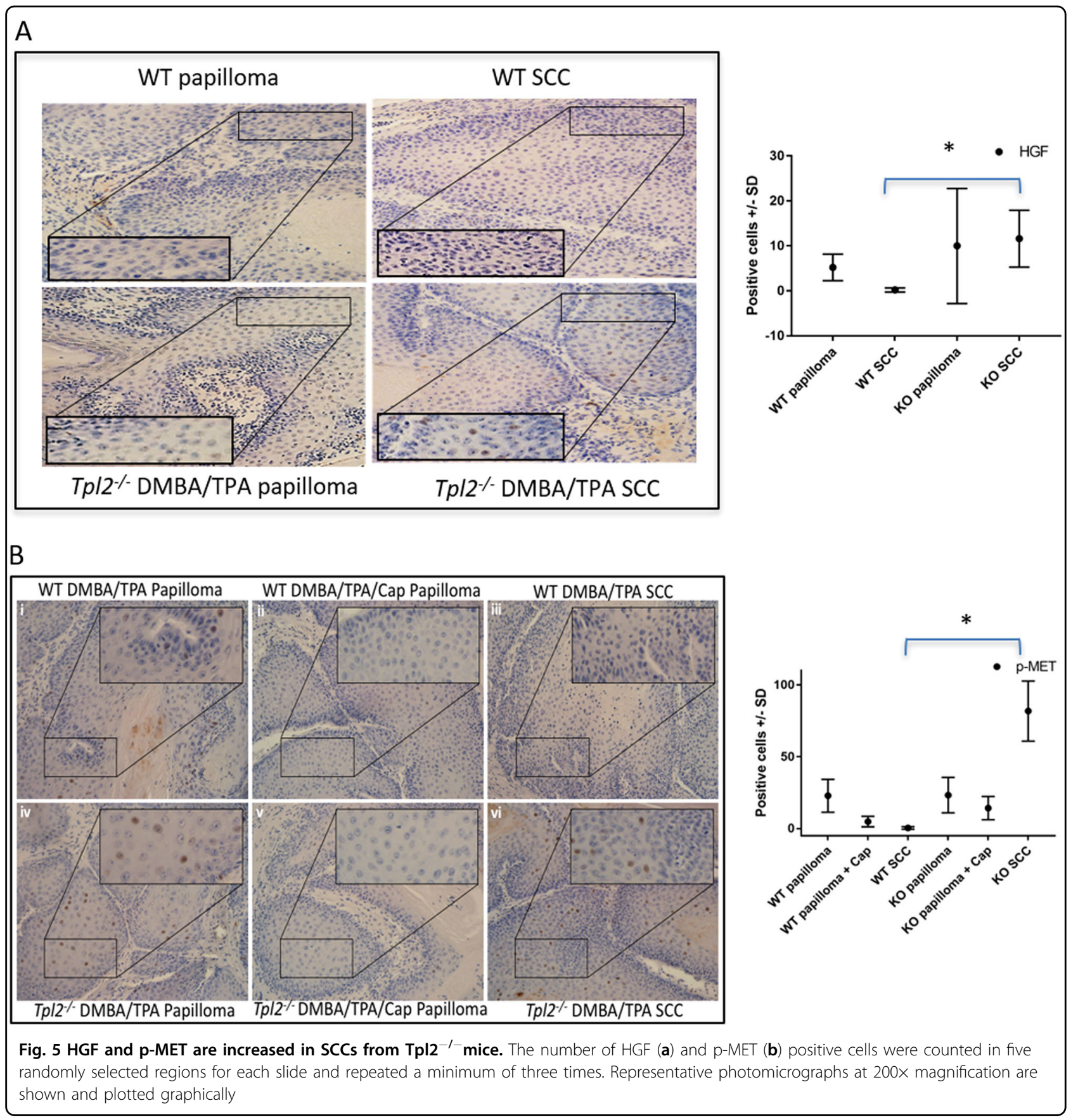

TGF $\beta$ is normally secreted by fibroblasts and carcinoma cells and can initiate multiple downstream signaling pathways including those mediated by SMAD proteins. In skin, the role of TGF $\beta$ is complicated and paradoxical ${ }^{36}$. Under normal conditions TGF $\beta$ is tumor suppressive by serving as a negative regulator of proliferation. Loss of TGF $\beta$ and/or its receptors or associated SMAD proteins is associated with tumorigenesis and an increased risk of malignant conversion ${ }^{41}$. However, as cancer advances and cells become refractory to the inhibitory effects of TGF $\beta$,
TGF $\beta$ can be tumor promoting by stimulating epithelial to mesenchymal transition (EMT), weakening immune surveillance, and enhancing tumor proliferation and chemoresistance $^{36}$. A wide body of literature has implicated RAS signaling in the TGF $\beta$ switch from tumor suppressor to tumor promoter, as RAS and TGF beta family members can crosstalk at several different levels ${ }^{42}$.

In this paper we found two-fold lower TGF $\beta 1$ protein levels in untreated keratinocytes from $T p l 2^{-1-}$ mice but no defect in TGF $\beta$ receptors or associated SMAD 
proteins. However, this defect doesn't appear to be responsible for the faster cell cycle (Fig. 3d) or the increased HGF (unpublished findings) in $T p l 2^{-1-}$ keratinocytes, as exogenous TGF $\beta 1$ could not reverse the effect.

In contrast to negative regulation of HGF by TGF $\beta$, multiple hormone responsive and transcription factor binding elements on the promoter of HGF can positively activate transcription of this growth factor ${ }^{43}$. Among the strongest inducers of HGF gene expression in fibroblasts are inflammatory mediators such as prostaglandin E2, synthesized by cyclooxygenase- 2 , and IL-1 $\beta^{44,45}$. Although we have yet to determine which, if any, factors are responsible for the upregulation of HGF in our system PGE2, COX- 2 and IL-1 $\beta$ have all been previously reported by our laboratory to be significantly elevated in $T p l 2^{-1-}$ keratinocytes and/or skin, and thus may be good candidates to explain this upregulation ${ }^{11,15}$.

In cancer, HGF binding to MET can be paracrine or autocrine, as HGF produced by stromal fibroblasts can bind to MET on neighboring cancer cells, or cancer cells can produce both HGF and MET. We found elevated pMET in $\mathrm{v}_{\text {-ras }}{ }^{\mathrm{Ha}}$-transduced keratinocytes, papillomas, and SCCs from Tpl2 ${ }^{-1-}$ mice. To assess whether pharmacological inhibition of MET could prevent skin tumorigenesis and/or progression in Tpl2 $2^{-1-}$ mice we conducted a DMBA/TPA skin carcinogenesis protocol. Capmatinib is a selective MET inhibitor currently in Phase I/II studies for a variety of cancers ${ }^{31,32}$. However, to the best of our knowledge it hasn't been studied in cutaneous SCC or a long-term mouse study. We found that capmatinib treatment for 52 weeks in both genotypes was well tolerated, as no adverse effects on body weight or overall health were found (data not shown). Capmatinib treatment reduced overall tumor burden by $60 \%$ in Tpl2 ${ }^{-1-}$ mice. More significant was the finding that all malignant conversion of benign papillomas to invasive SCC in $T p l 2^{-1-}$ mice was prevented in mice fed a capmatinib diet, suggesting MET plays an integral role in skin cancer progression. In both genotypes, more males developed tumors than female mice, but $T p l 2^{-1-}$ males responded better to capmatinib. However, since sex differences were not the major objective of this work, estrus cycles in female mice were not synchronized and thus could have influenced individual variations between females ${ }^{46}$.

Recent evidence suggests that EGFR is an essential factor for MET-driven skin carcinogenesis ${ }^{20}$. In the presence of a strong promoter, MET activation in the absence of Hras mutations is sufficient to drive skin tumorigenesis ${ }^{20}$. However, MET-driven tumors regress when EGFR is blocked. We add support to the notion that MET and EGFR cooperate in skin carcinogenesis, as capmatinib treatment could block the HGF-stimulated upregulation in p-EGFR.
One of the more interesting results we found in this study was that $89 \%$ of $T p l 2^{-1-}$ mice treated solely with the carcinogen DMBA developed papillomas, all of which stayed small and didn't undergo malignant conversion. As expected no WT mice receiving DMBA alone developed papillomas. The Hras gene is a major target of DMBA ${ }^{2}$. Typically, a pro-inflammatory tumor promoter such as TPA is necessary to induce tumorigenesis. Mechanistically, tumor promoters work by inducing a number of inflammatory factors including IL-1 alpha, COX-2, PGE2, and EP2. These factors play a central role in promotion, as DMBA/TPA induced tumor incidence and progression is lower in mice devoid of these genes ${ }^{47-49}$. We have previously reported that skin from $T p l 2^{-1-}$ mice have increased epidermal thickness marked by significantly higher levels of neutrophils, macrophages, prostaglandins/ prostaglandin receptors, and pro-inflammatory cytokines $^{11,15}$. It is likely that the elevated inflammatory state in Tpl2 ${ }^{-1-}$ mice can compensate for a promoter, as DMBA only was sufficient for tumor formation. However, the mechanism by which this occurs is still under investigation.

In summary, we have identified the HGF/MET pathway as critical to skin tumor growth and malignant conversion in $T p l 2^{-l-}$ mice. This provides new insight into how the MAPK pathway interacts with HGF/MET signaling and highlights the potential of MET inhibitors as therapeutic targets for cutaneous SCC.

\section{Materials and methods}

\section{Wild type and transgenic mice}

Male and female wild type $\left(T p l 2^{+/+}\right)$and knockout $\left(T p l 2^{-/-}\right) \mathrm{C} 57 \mathrm{Bl} / 6$ mice were engineered as previously described $^{4}$. All mice were bred and maintained at The American University animal facility (Washington, DC) under NIH guidelines and an approved IACUC protocol. $T p l 2^{-/-}$status was regularly confirmed by PCR.

\section{Primary keratinocyte isolation and treatment}

Primary keratinocytes and fibroblasts were isolated from $T p l 2^{-/-}$and WT $\left(T p l 2^{+/+}\right)$mice pups at $1-3$ days of age according to standard protocols ${ }^{50}$. Keratinocytes were grown in PromoCell keratinocyte growth media (VWR, Philadelphia, PA) containing hormone supplements, Penicillin-Streptomycin $(10,000 \mathrm{U} / \mathrm{ml})$ and low $(0.05 \mathrm{mM})$ calcium. Fibroblasts were maintained in DMEM containing 10\% FBS, Penicillin-Streptomycin $(10,000 \mathrm{U} / \mathrm{ml})$ and Glutamax $(200 \mathrm{mM})$. For some experiments keratinocytes were infected with $\mathrm{v}$-ras ${ }^{\mathrm{Ha}}$ retrovirus as described elsewhere ${ }^{50}$. For in vitro capmatinib studies keratinocytes were treated with $0.1 \mathrm{nM}$ capmatinib (INCB28060; Selleck Chemicals, Houston, TX) for $2 \mathrm{~h}$ followed by application of $20 \mathrm{ng} / \mathrm{mL}$ HGF or vehicle (HGF; R\&D Systems, Minneapolis, MN) for 
Table 2 List of primers for real-time PCR

\begin{tabular}{llll}
\hline Target & Forward sequence & Reverse sequence & Amplicon length \\
\hline Tgfb1 & CTCCCGTGGCTCTAGTGC & GCCTAGTTTGGACAGGATCTG & 133 \\
Tgfrl & TCTGCATTGCACTTATGCTGA & AAAGGGCGATCTAGTGATGGA & 100 \\
Tgfr2 & CCGCTGCATATCGTCCTGTG & AGTGGATGGATGGTCCTATTACA & 131 \\
HGF & CCTGACACCACTTGGGAGTA & CTTCTCCTTGGCCTTGAATG & 91 \\
MET & CATTTTACGGACCCAACCA & TGTCCGATACTCGTCACTGC & 74 \\
Fas & GCGGGTTCGTGAAACTGATAA & GCAAAATGGGCCTCCTTGATA & 61 \\
B-actin & GGCTACAGCTTCACCACCAC & ATGCCACAGGATTCCATACC & 214 \\
\hline
\end{tabular}

$15 \mathrm{~min}$ or $24 \mathrm{~h}$ prior to protein isolation. For conditioned media experiments, media was collected from treated keratinocytes or fibroblasts $18 \mathrm{~h}$ post treatment, centrifuged, and normalized to cell number.

\section{Quantitative polymerase chain reaction (qPCR)}

Total RNA was extracted and purified from cultured primary keratinocytes or fibroblasts using RNeasy Mini kit according to manufacturer's instructions (Qiagen, Germantown, MD). Complementary DNA (cDNA) was reverse transcribed from template RNA and qPCR was performed using primers obtained from Harvard Primer Bank (Table 2) as previously described ${ }^{40}$. All experiments were repeated a minimum of three times.

\section{Western blotting}

Total protein lysates were prepared from keratinocytes and fibroblasts using RIPA buffer containing Halt protease/phosphatase inhibitors in accordance with the manufacturer's protocol (Thermo Fisher Scientific, Rockford, IL, USA). Twenty-five micrograms of protein was electrophoresed using 4-12\% gradient SDSpolyacrylamide gels, transferred to PVDF membrane and blocked with 5\% BSA. Primary antibodies for HGF (Abcam; cat \#ab83760), TGF $\beta$ (Abcam; cat \#ab92486), TGFßR1 (Abcam; ab31013), TGFßR2 (Abcam; ab186838), SMAD2 (Abcam; ab63576), SMAD4 (Abcam; ab236321) MET (Cell Signaling; cat \#3127), p-MET (Abcam; ab5662), GAPDH (Cell Signaling, Cat \#5174), B-actin (Cell Signaling; Cat \#4970), tubulin (Cell Signaling, Cat \#15115) were incubated with membranes at 1:1000. Antirabbit HRP secondary antibodies (Cell Signaling Technology), followed by West Dura Chemilluminescent substrate (Thermo, Rockland, IL) were used for signal detection. Bands were quantified using NIH Image J and normalized to the densitometry for the respective housekeeping gene. All Western blots used pooled protein from triplicate samples and were repeated a minimum of three times. Quantification of Western blots is displayed in Supplementary data A.

\section{WST-1 cell proliferation assay}

$5 \times 10^{4} \mathrm{v}-$ ras $^{\mathrm{Ha}}$-transduced WT or Tpl $2^{-1-}$ keratinocytes were plated in quadruplicate in hormone supplemented media or conditioned media from WT or Tpl2 -I- fibroblasts and incubated at $37{ }^{\circ} \mathrm{C}$ in $5 \% \mathrm{CO}_{2}$. After $24 \mathrm{~h}$, WST-1 was added to each well, incubated, and absorbance read on a microplate reader at $450 \mathrm{~nm}$. Results were normalized to the WT keratinocyte control. For some experiments keratinocyte media was supplemented with $10 \mathrm{ng} / \mathrm{mL}$ TGF $\beta$ for $24 \mathrm{~h}$.

\section{Immunohistochemistry}

Immunohistochemistry was performed as previously described $^{15}$. Formalin-fixed, paraffin-embedded skin and tumor sections from $\mathrm{Tpl} 2^{-1-}$ and WT mice were processed into paraffin blocks from which $4 \mu \mathrm{m}$ sections were cut and stained with hematoxylin and eosin (H\&E).

Primary antibodies for HGF and p-MET (Abcam; Cambridge, MA, cat \# ab83760 and ab5662) and antirabbit secondary antibodies (Cell Signaling; Danvers, MA, cat \#7074 S) were used. Sections came from a minimum of three individual mice per treatment. As our in vivo experiment only produced 1 SCC in WT mice, we included three additional WT SCC sections from a parallel in vivo study (using the same dosage and timing of DMBA/TPA administration) to increase the number of WT SCCs used for immunohistochemistry. Representative areas were photographed at $200 \times$ magnification. For p-MET and HGF staining in papillomas and squamous cell carcinomas the number of positive cells for each section were manually counted in five microscopic fields. For HGF staining in the skin, the optical density (OD) of HGF was quantified using FIJI Image J.

\section{Zymography}

Zymography was performed as previously reported ${ }^{14}$. Briefly, harvested cell-free conditioned media was electrophoresed on $10 \%$ tris-glycine gels containing $0.1 \%$ gelatin. Zymogram gels were incubated in zymogram renaturing buffer, developing buffer, stained with 
Coomassie blue G-250, and destained in deionized water. Bands were quantified using NIH Image J.

\section{In vitro conversion assay}

An in vitro conversion assay was modified from Morgan, et al. ${ }^{51}$. Fresh primary keratinocytes were plated in lowcalcium $(0.05 \mathrm{mM})$ keratinocyte media and grown to confluence. Cells were v-ras ${ }^{\mathrm{Ha}}$-transfected for $48 \mathrm{~h}$ and transferred to low-calcium keratinocyte media. After 2 weeks cell cultures were maintained in either $0.5 \mathrm{mM}$ high calcium (HiCa) media, $\mathrm{HiCa} T p l 2^{+/+}$fibroblast conditioned media, or $\mathrm{HiCa} T p l 2^{-1-}$ fibroblast conditioned media for 8 weeks while proliferative foci formed. Media was changed twice weekly. At termination, the keratinocyte cultures were stained and fixed with $0.35 \%$ rhodamine in formalin, then imaged with a fluorescence microscope.

\section{Tumor induction experiments}

A total of $25 \mathrm{Tpl} 2^{-1-}$ mice and $29 \mathrm{C} 57 \mathrm{BL} / 6 \mathrm{WT}$ 8-10 week old mice were initiated with 7,12-dimethylbenz(a)anthracene (DMBA; $100 \mu \mathrm{g} / 200 \mu \mathrm{l}$ acetone) on shaved right dorsal skin. Of these mice, 9/genotype received no further treatment (identified as DMBA only mice). For the remaining sixteen $T p l 2^{-/-}$mice and twenty WT mice, promotion began 1 week later with twice weekly application of TPA painted on the skin $(10 \mu \mathrm{g} / 200 \mu \mathrm{l}$ acetone) and continued for 20 additional weeks. Mice were maintained on AIN-93M diet $\left(T p l 2^{-1-}\right.$; $n=8$ or WT; $n=12)$ or AIN-93M diet containing $44 \mathrm{mg} /$ kg capmatinib ( $n=8$ /genotype), a selective c-MET inhibitor. Two additional control groups/genotype received only acetone $(n=2)$ or TPA $(n=3)$. The number and size of tumors were recorded weekly. The numbers of mice required for this experiment are the minimum numbers needed to achieve statistical power. All groups were matched for age, weight and sex. Tumor-bearing animals were individually housed to avoid injury to the tumor sites. Animals were euthanized 52 weeks after the date of initiation, or at an earlier time point if the animal was deemed moribund by the veterinary staff. Portions of skin and tumors were either snap frozen for DNA/RNA/protein isolation or formalin-fixed for IHC. All tumors underwent a histological examination in a blinded fashion by a certified pathologist to determine tumor type (Mass Histology Services, Worcester, MA).

\section{Statistical analyses}

Data was tested for normality, model assumptions were checked, and the data were analyzed with SPSS software. Tumor induction experiments, in vitro conversion assays, zymography, and Western blotting examining genotype and drug effects were analyzed through two-way ANOVA with Tukey's post hoc test. One-way ANOVA using Fisher's least significant difference (LSD) post-hoc test was used to analyze qPCR, IHC on skin sections, FAS expression, Western blots that looked at genotype only effects, and viability experiments. Significance for all analyses was assumed at a $p$-value of 0.05 or less. Significance values of $p \leq 0.05$ are indicated in figures with a single asterisk (*), $p \leq 0.01$ with a double asterisk $(* *)$, and $p \leq 0.001$ with a triple asterisk ${ }^{(* * * *)}$.

\section{Acknowledgements}

This work was supported by National Institutes of Health (2R15CA152907-02 and 1UA5CA152907-01A1).

\section{Author Contributions}

K.D. initiated the project. L.F., N.B., and K.D. designed the animal experiments and L.F., N.B., K.K., D.K., and K.D. performed the animal experiments and analyzed data. K.F., E.M., N.B., L.F., J.C.G., K.D., and D.K. designed in vitro experiments, performed the experiments and analyzed data. N.B. and K.D. wrote the manuscript.

\section{Conflict of interest}

The authors declare that they have no conflict of interest.

\section{Publisher's note}

Springer Nature remains neutral with regard to jurisdictional claims in published maps and institutional affiliations.

Supplementary Information accompanies this paper at (https://doi.org/ 10.1038/s41389-018-0109-8).

Received: 11 August 2018 Revised: 16 November 2018 Accepted: 3 December 2018

Published online: 04 January 2019

\section{References}

1. Karia, P. S., Han, J. \& Schmults, C. D. Cutaneous squamous cell carcinoma: estimated incidence of disease, nodal metastasis, and deaths from disease in the United States, 2012. J. Am. Acad. Dermatol. 68, 957-966 (2013).

2. Abel, E. L., Angel, J. M., Kiguchi, K. \& DiGiovanni, J. Multi-stage chemical carcinogenesis in mouse skin: fundamentals and applications. Nat. Protoc. 4, 1350-1362 (2009)

3. DeCicco-Skinner, K., Deshpande, M. \& Wiest, J. The role of Tpl2 protein kinase in carcinogenesis and inflammation, Advances in Protein Kinases, Dr. Gabriela Da Silva Xavier (Ed.), ISBN:978-953-51-0633-3, INTECH Open Access Publisher, 2012. https://www.intechopen.com/books/advances-in-protein-kinases/therole-of-tpl2-in-carcinogenesis-and-inflammation. Accessed 20 December 2012.

4. Ceci, J. D. et al. Tpl-2 is an oncogenic kinase that is activated by carboxyterminal truncation. Genes Dev. 11, 688-700 (1997).

5. Salmeron, A. et al. Activation of MEK-1 and SEK-1 by Tpl-2 proto-oncoprotein, a novel MAP kinase kinase kinase. EMBO J. 15, 817-826 (1996).

6. Sounvinos, G., Tsatsanis, C. \& Spandidos, D. A. Overexpression of the Tpl-2/Cot oncogene in human breast cancer. Oncogene 18, 4968-4973 (1999).

7. Eliopoulos, A. G. et al. The oncogenic protein kinase Tpl-2/Cot contributes to Epstein-Barr virus-encoded latent infection membrane protein 1-induced NFkappaB signaling downstream of TRAF2. J. Virol. 76, 4567-4579 (2002).

8. Christoforidou, A. V., Papadaki, H. A., Margioris, A. N., Eliopoulos, G. D. \& Tsatsanis, C. Expression of the Tpl2/Cot oncogene in human T-cell neoplasias. Mol. Cancer 3, 34 (2004).

9. Lee, J. H. et al. TPL2 is an oncogenic driver in keratocanthoma and squamous cell carcinoma. Cancer Res. 76, 6712-6722 (2016).

10. Tsatsanis, C. et al. Tpl2 and ERK transduce antiproliferative T cell receptor signals and inhibit transformation of chronically stimulated T cells. Proc. Natl Acad. Sci. USA 105, 2987-2992 (2008).

11. Decicco-Skinner, K. L., Trovato, E. L., Simmons, J. K, Lepage, P. K. \& Wiest, J. S. Loss of tumor progression locus 2 (tpl2) enhances tumorigenesis and inflammation in two-stage skin carcinogenesis. Oncogene 30, 389-397 (2011). 
12. Koliaraki, V., Roulis, M. \& Kollias, G. Tpl2 regulates intestinal myofibroblast HGF release to suppress colitis-associated tumorigenesis. J. Clin. Invest. 122, 4231-4242 (2012).

13. Gkirtzimanaki, K. et al. TPL2 kinase is a suppressor of lung carcinogenesis. Proc. Natl Acad. Sci. USA 110, E1470-E1479 (2013).

14. DeCicco-Skinner, K. et al. Tpl2 knockout keratinocytes have increased biomarkers for invasion and metastasis. Carcinogenesis 34, 2789-2798 (2013).

15. DeCicco-Skinner, K. L. et al. Altered prostanoid signaling contributes to increased skin tumorigenesis in Tpl2 knockout mice. PLOS ONE 8, e56212 (2013).

16. De Silva, D. M. et al. Targeting the hepatocyte growth factor/Met pathway in cancer. Biochem. Soc. Trans. 45, 855-870 (2017).

17. Matsumoto, K., Umitsu, M., De Silva, D. M., Roy, A. \& Bottaro, D. P. Hepatocyte growth factor/MET in cancer progression and biomarker discovery. Cancer Sci. 108, 296-307 (2017).

18. Lee, Y. J. et al. Expression of the c-Met proteins in malignant skin cancers. Ann. Dermatol. 23, 33-38 (2011).

19. Szabo, R. et al. c-Met-induced epithelial carcinogenesis is initiated by the serine protease matriptase. Oncogene 30, 2003-2016 (2011).

20. Cataisson, C. et al. MET signaling in keratinocytes activates EGFR and initiates squamous carcinogenesis. Sci. Signal 9, ra62 (2016).

21. Graveel, C. R., Tolbert, D. \& Vande Woude, G. F. MET: a critical player in tumorigenesis and therapeutic target. Cold Spring Harbor Perspect. Biol 5, a009209 (2013) https://doi.org/10.1101/cshperspect.a009209. Accessed 20 December 2018

22. Sharma, N. \& Adjei, A. A. In the clinic: ongoing clinical trials evaluating c-METinhibiting drugs. Ther. Adv. Med. Oncol. 3(1 Suppl), S37-S50 (2011).

23. Liu, X. et al. A novel kinase inhibitor, INCB28060, blocks C-MET-dependent signaling, neoplastic activities, and cross-talk with EGFR and HER-3. Clin. Cancer Res. 17, 7127-7138 (2011).

24. Lara, M. S. et al. Preclinical evaluation of MET inhibitor INC-280 with or without the epidermal growth factor receptor inhibitor Erlotinib in non-small-cell lung cancer. Clin. Lung. Cancer 18, 281-285 (2017).

25. Imura, Y. et al. Functional and therapeutic relevance of hepatocyte growth factor/c-MET signaling in synovial sarcoma. Cancer Sci. 107, 1867-1876 (2016).

26. Han, P. et al. Dual inhibition of Akt and c-Met as a second-line therapy following acquired resistance to sorafenib in hepatocellular carcinoma cells. Mol. Oncol. 11, 320-334 (2017).

27. Jia, Y. et al. EGF816 exerts anticancer effects in non-small cell lung cancer by irreversibly and selectively targeting primary and acquired activating mutations in the EGF receptor. Cancer Res. 76, 1591-1602 (2016).

28. Brandes, F. et al. Targeting CMET with INC280 impairs tumour growth and improves efficacy of gemcitabine in a pancreatic cancer model. Bmc. Cancer 15, 71 (2015)

29. Imura, Y. et al. Combined targeting of mTOR and c-MET signaling pathways for effective management of epithelioid sarcoma. Mol. Cancer 13, 185 (2014).

30. Krepler, C. et al. Personalized preclinical trials in BRAF inhibitor-resistant patientderived xenograft models identify second-line combination therapies. Clin. Cancer Res. 22, 1592-1602 (2016).

31. Qi, X.-S., Guo, X.-Z., Han, G.-H., Li, H.-Y. \& Chen, J. MET inhibitors for treatment of advanced hepatocellular carcinoma: a review. World J. Gastroenterol.: Wjg. 21, 5445-5453 (2015)

32. Bauer, T. M. et al. MINI01.03: Phase (Ph) I Study of the Safety and Efficacy of the CMET Inhibitor Capmatinib (INC280) in Patients with Advanced cMET+NSCLC: Topic: Medical Oncology. J. Thorac. Oncol. 11(11s), S257-s8 (2016).
33. Ishikawa, T. et al. Hepatocyte growth factor/c-met signaling is required for stem-cell-mediated liver regeneration in mice. Hepatology 55, 1215-1226 (2012).

34. Zhao, L. et al. c-Met identifies a population of matrix metalloproteinase 9producing monocytes in peritumoural stroma of hepatocellular carcinoma. J. Pathol. 237, 319-329 (2015).

35. Cheng, N., Chytil, A., Shyr, Y., Joly, A. \& Moses, H. L. Transforming growth factorbeta signaling-deficient fibroblasts enhance hepatocyte growth factor signaling in mammary carcinoma cells to promote scattering and invasion. Mol. Cancer Res.: Mcr. 6, 1521-1533 (2008).

36. Colak, S. \& Ten Dijke, P. Targeting TGF-beta Signaling in Cancer. Trends Cancer 3, 56-71 (2017)

37. Johannessen, C. M. et al. COT drives resistance to RAF inhibition through MAP kinase pathway reactivation. Nature 468, 968-972 (2010).

38. Flaherty, K. T. et al. Combined BRAF and MEK inhibition in melanoma with BRAF V600 mutations. N. Eng. J. Med. 367, 1694-1703 (2012).

39. Koh, S. A. \& Lee, K. H. HGF mediated upregulation of lipocalin 2 regulates MMP9 through nuclear factor-kappaB activation. Oncol. Rep. 34, 2179-2187 (2015).

40. Decicco-Skinner, K. L. et al. Tpl2 knockout keratinocytes have increased biomarkers for invasion and metastasis. Carcinogenesis 34, 2789-2798 (2013).

41. Glick, A. B. The Role of TGFbeta Signaling in Squamous Cell Cancer: Lessons from Mouse Models. J. Skin. Cancer 2012, 249063 (2012).

42. Grusch, $M$. et al. The crosstalk of RAS with the TGF- $\beta$ family during carcinoma progression and its implications for targeted cancer therapy. Curr. Cancer Drug. Targets 10, 849-857 (2010)

43. Liu, Y., Michalopoulos, G. K. \& Zarnegar, R. Structural and functional characterization of the mouse hepatocyte growth factor gene promoter. J. Biol. Chem. 269, 4152-4160 (1994).

44. Matsumoto, K., Okazaki, H. \& Nakamura, T. Novel function of prostaglandins as inducers of gene expression of HGF and putative mediators of tissue regeneration. J. Biochem. 117, 458-464 (1995).

45. Matsumoto, K., Tajima, H., Okazaki, H. \& Nakamura, T. Negative regulation of hepatocyte growth factor gene expression in human lung fibroblasts and leukemic cells by transforming growth factor-beta 1 and glucocorticoids. J. Biol. Chem. 267, 24917-24920 (1992).

46. Wood, P. A. \& Hrushesky, W. J. Sex cycle modulates cancer growth. Breast Cancer Res. Treat. 91, 95-102 (2005).

47. Apte, R. N. et al. Effects of micro-environment- and malignant cell-derived interleukin-1 in carcinogenesis, tumour invasiveness and tumour-host interactions. Eur. J. Cancer 42, 751-759 (2006).

48. Tiano, H. F. et al. Deficiency of either cyclooxygenase (COX)-1 or COX-2 alters epidermal differentiation and reduces mouse skin tumorigenesis. Cancer Res. 62, 3395-3401 (2002)

49. Sung, Y. M., He, G. \& Fischer, S. M. Lack of expression of the EP2 but not EP3 receptor for prostaglandin E2 results in suppression of skin tumor development. Cancer Res. 65, 9304-9311 (2005).

50. Lichti, U., Anders, J. \& Yuspa, S. H. Isolation and short-term culture of primary keratinocytes, hair follicle populations and dermal cells from newborn mice and keratinocytes from adult mice for in vitro analysis and for grafting to immunodeficient mice. Nat. Protoc. 3, 799-810 (2008).

51. Morgan, D. et al. Development of an in vitro model to study carcinogeninduced neoplastic progression of initiated mouse epidermal cells. Cancer Res. 52, 3145-3156 (1992) 